\title{
Performance Evaluation of DCT And Wavelet Coding of Image Transmission By Using of DM Technique Over Topical Shallow-Water Environment
}

\author{
Sendi Eka Nilasari, I Gede Puja Astawa, Tri Budi Santoso
}

Telecommunication Study Program, Politeknik Elektronika Negeri Surabaya

Jl. Raya ITS, Sukoliko, +62-31 5947280

sendi@student.eepis-its.edu, tribudi@eepis-its.edu, puja@eepis-its.edu

\begin{abstract}
Image transmission over under water acoustic channel is one of research trends that were developed to support under ocean environment monitoring. The result of study about the source coding performance for image transmission over underwater acoustic channel over tropical shallow-water environment is presented in this paper. By using the discrete cosine transform (DCT) and wavelet coding, image file was compressed and converted into binary data sequence. Transmission process was conducted with multicarrier OFDM system over under water acoustic channel. An evaluation has been implemented on base band scale by adopting channel model of previous research. With the DCT technique at Eb/No $20 \mathrm{~dB}$ was achieved the value of PNSR $12.69 \mathrm{~dB}$, and bit error 0.0025 . While by using the wavelet technique, at same $\mathrm{Eb} /$ No value, achieved the value of PSNR at $21.38 \mathrm{~dB}$ and bit error rate 0.0022 . The performance evaluation also conducted visually and showing similar trend as simulation result by using pseudo random data.
\end{abstract}

Keywords: DCT, wavelet, OFDM, underwater acoustic.

\section{INTRODUCTION}

At the last three decades it has been seen the development of underwater acoustic communication in many application as study of marine, oceanography, oil exploration in the off coast area and defense system. Next researches in the last few years have been able to increase performance and toughness system than previous existing [1]. In this paper it is also described the direction of development of communication underwater acoustic research. 
Characterization of the acoustic propagation channel nature underwater by using statistical approaches to data measurement results have been proposed [2], [3], [4] and [5]. In this case, the condition of the acoustic channel with variety depth, starts from shallow conditions until the relative conditions inside. A model of the acoustic channel using ray tracing approach was presented to the environment of the Persian coast [2] and [3] The paper is complemented by transmission information testing with QPSK. Citre in her paper report [4] presents the characterization of the acoustic channel for medium-range on a shallow water conditions. The other research for the characterization of the acoustic channel shallow conditions have been presented by Borowski [5].Here a modeling of acoustic channel presented for coast environmental and the estuary of the coast and equipped by testing over the simulation of pass band with software. In general, model channel used at modeling paper-paper above is a condition of shallow waters on the environment tropical, so it is not relating to tropical shallow-water.

An efficient compression model in the process of image coding for the environment underwater has been proposed [6]. Here a coding scheme by utilizing wavelet as preprocessing applied to deprive of spatial redundancy on visual information, and performed with adopt algorithm of wavelet treebased wavelet difference reduction. This technique is being able to give a reliable performance, but on paper is not directed at process of shipping image to remote area, and limited at process of compression.

The model of image transmission over underwater acoustic channel has been presented at paper [7] [8], and [9]. At papers above, lot of process of image compression and sending image method are efficient when using acoustic media underwater. Here, the compression process is forwarded to sending over communication multicarrier system and evaluated performance of error rate. However, generally on the papers, it does not specifically describe ocean environment where system will be tested.

In this paper it is described the study about the influence of choosing channel coding for transmission system multi carrier orthogonal frequency division multiplexing (OFDM) at acoustic channel underwater in ocean environment tropical shallow-water. By utilizing the result of propagation characterization that achieved at previous research [10], has been obtained description of value of noise environment, fading, and power delay profile. Furthermore, it has been arranged a base band transmission model to evaluate source coding technique DCT and wavelet by utilizing transmission system OFDM. The performance of both coding technique will be evaluated over software simulation, which in this case is error rate performance and comparing visual achievement from transmitted image. One contribution in this paper is model of image transmission system conducted using parameters which present the condition of swallow-water environment in tropical area.

In addition, the paper is organized as follows. At the second chapter will be presented acoustic channel underwater and propagation parameter 
obtained over previous study. Coding and image transmission models will be presented at the third chapter. The performance evaluation of coding technique will be shown at the fourth chapter. In the fifth chapter, will describe the conclusion and the next research activity.

\section{CHARACTERISTIC OF UNDERWATER ACOUSTIC CHANNEL AT SWALLOW-WATER}

Sound propagation from the source to the receiver can be described in the form of a series of different path related to transmission from image sources (sources of shadows) which is formed geometrically from the reflection to surface and base

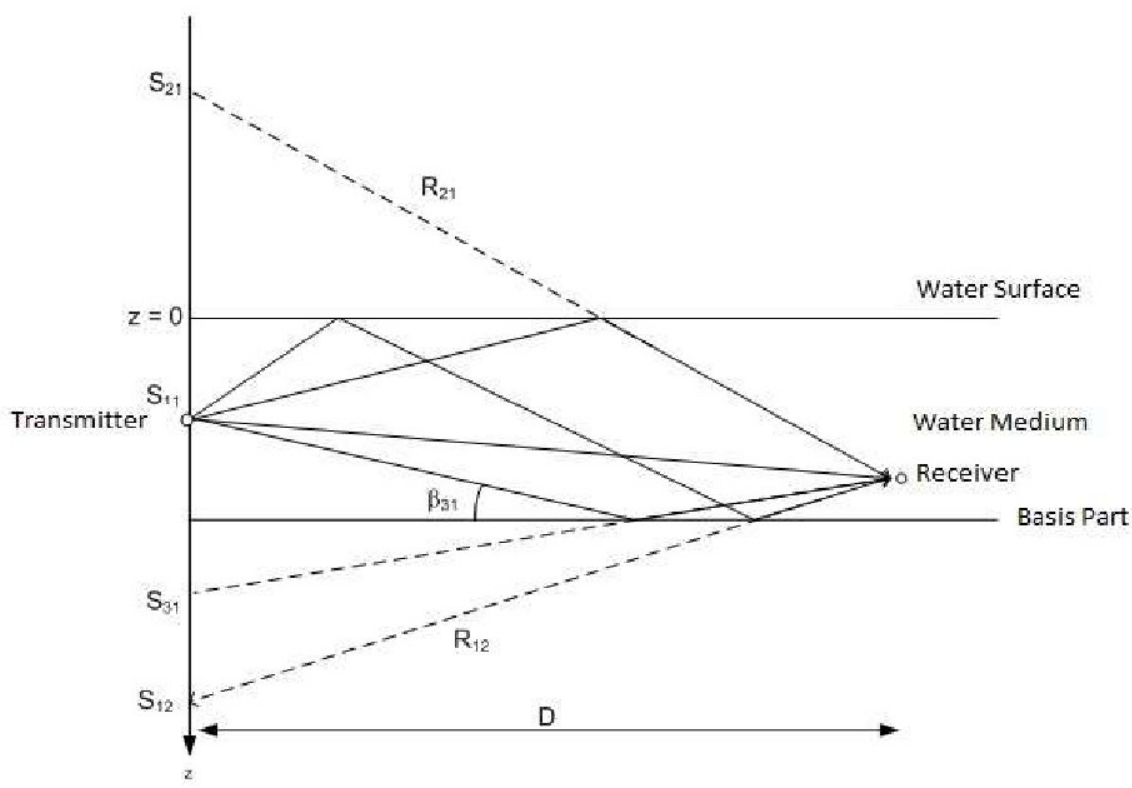

Figure 1. Propagation of underwater acoustic channel in the swallow-water

If $S(t)$ is transmitted signal, each shadow source can contribute in a form of delayed replication that are affected by losses of transmission and accumulation of coefficient of reflection all the surface took place. In simple terms can be expressed with the following formula.

$$
p_{j i}(t)=S\left(t-\tau_{j i}\right) A_{j i}
$$

Where $A_{j i}$ are accumulation rate of losses transmission at path $j i$, and $\tau_{j i}$ is rate of delayed propagation. For simplification of surface stated reflection surface coefficient $V_{s}$ valued 1 and has a change phase as $\pi$ radiant and base reflection coefficient $V_{b}$ valued without any change of phase.

\subsection{Underwater Acoustic Channel Characteristic}

Characterization of the acoustic channel has done with measurement of propagation characteristics on a standard test of a towing tank with dimensions: width $12 \mathrm{~m}$, length $200 \mathrm{~m}$, and $6 \mathrm{~m}$ depth. Chirp signal standard, 
sinus and pulses are emitted from an underwater speaker and hydrophone array was caught with a position of $80,100,120 \sim 180 \mathrm{~m}$ from the source. The data processing is done over benchmarking measurement results with a standard channel model has been widely used in communication systems. It is presented in a simple block diagram as in Figure 2.

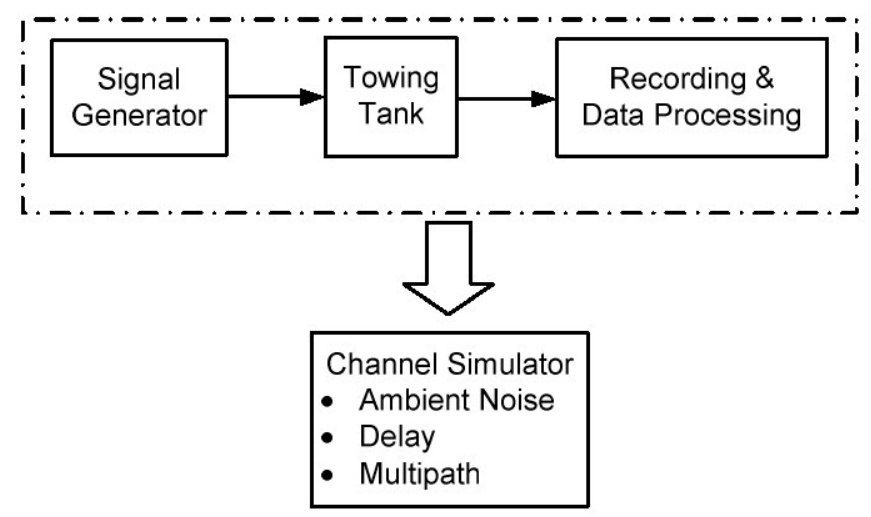

Figure 2. Characterization of propagation parameter

\subsection{The Results of Channel Propagation Parameter Characterization}

Characterization of noise environmental was conducted over analysis of probability density function (pdf), measurement data conducted by utilizing technique of maximum likelihood estimation (MLE). Results obtained give a sense that signal of noise environment approaching the nature of signals random distributed Gaussian with the average value of zero and variant 0,028 . Comparison probability density function (pdf), data of measurement result of noise environment and pdf Gaussian. This can be seen clearly as in a picture $3 \mathrm{a}$.

Probability density distribution form (pdf) working with the frequency $4000 \mathrm{~Hz}$ with the distance between transmitter and receiver is within a range from $80 \mathrm{~m}$ to $120 \mathrm{~m}$. With approach of MLE obtained results that pdf pattern has similarities to Log normal distribution, the mean value 1,028 and variance 1.35. Compared to distribution of Rayleigh, form of pdf data obtained have different patterns. It can be seen more clearly as in Figure $3 \mathrm{~b}$ 


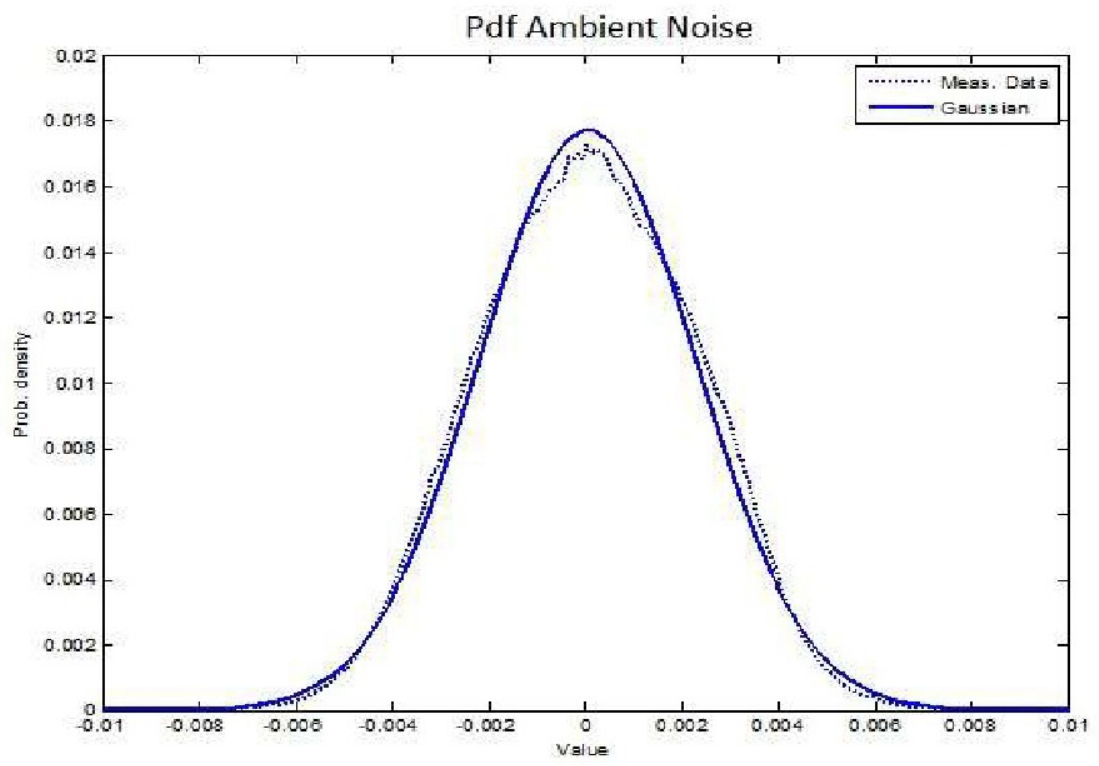

Figure 3a. Pdf noise Environment

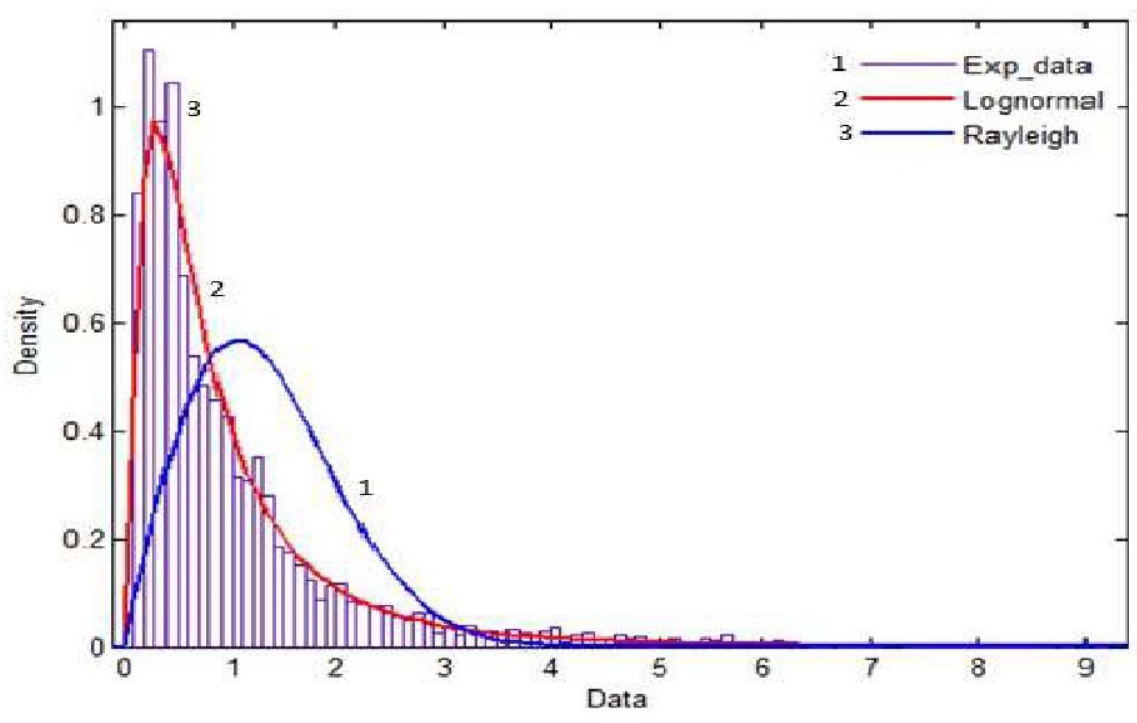

Figure 3b. Pdf fading at channel

\section{MODEL OF IMAGE TRANSMISSION WITH DCT DAN WAVELET CODING}

Several stages simulation of system OFDM will be divided into three namely include: the transmitter (Tx), channel (underwater), and the receiver $(\mathrm{Rx})$. Data input is a form of images that have been over the process of RGB to grey. The next step is compression and conversion to form a row of bit-bit binary using the technique of source coding, which in this case is the DCT and Wavelet. 
The general form of DCT Transformation for two dimensions can be expressed in the form of the following equation:

$$
\begin{gathered}
C(u, v)=\alpha(u) \alpha(v) \sum_{x=0}^{N-1} \sum_{y=0}^{N-1} f(x, y) \cos \left[\frac{\pi(2 x+1) u}{2 N}\right] \cos \left[\frac{\pi(2 y+1) v}{2 N}\right] \\
\alpha(u)=\left\{\begin{array}{lll}
\sqrt{\frac{1}{N}} & \text { untuk } u=0 \\
\sqrt{\frac{2}{N}} & \text { untuk } u \neq 0
\end{array}\right.
\end{gathered}
$$

Where $C(u, v)$ is the point coordinates of a matrix has undergo a transformation DCT 2 dimension. $\mathrm{M}$ and $\mathrm{N}$ are many columns and line. Matrix image of Bunaken _ 010.jpg in gray scale divided into blocks 8x8, where each block will be transformed from the domain of spatial (distances) into domain frequency. On transforming wavelet, matrix image 'Bunaken _ 010.jpg' still within domains spatial. For converting process Discrete Wavelet Transform (DWT) 2 dimensions. Next process can be explained ass following equation.

$$
\begin{aligned}
f(x, y)=\frac{1}{\sqrt{M N}} & \sum_{m} \sum_{n} W_{\phi}\left(j_{0}, m, n\right) \phi_{j_{0} m, n}(x, y) \\
& +\frac{1}{\sqrt{M N}} \sum_{i=H, V, D} \sum_{j=j 0}^{\infty} \sum_{m} \sum_{n} W_{\psi}^{i}(j, m, n) \phi_{j_{0} m, n}^{i}(x, y)
\end{aligned}
$$

After going over a series of source coding process, the result is the bits that have been compressed. The series of binary in-mapper (modulation) using a digital modulation Binary Phase Shift Keying (BPSK). The results of modulation converted into serial to parallel so have a low data rate. At this stage, the pilot symbol is inserted (guard interval) purposed to Inter Carrier Interference (ICI) can be avoided. After parallel to serial conversion, it has been given addition of guard channels with utilizing cyclic prefix technique. This is done in order to anticipate the effects of inter symbol interference which caused by the influence of delay spread channel. The transmission process can be seen in Figure 4. While OFDM simulation parameters refer to Table 1. 


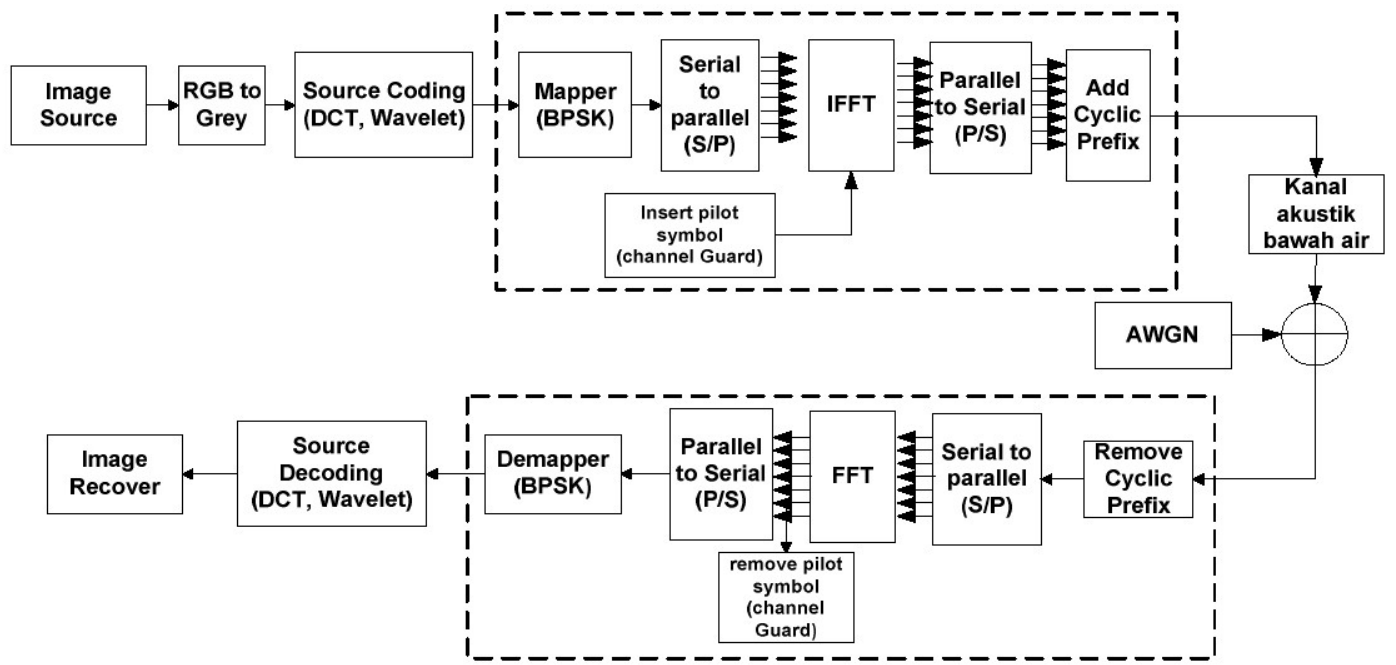

Figure 4. Block diagram of image transmission system

The output of OFDM transmitter can be expressed in the form of the following equation:

$$
S(n)=\sum_{k=0}^{N-1} X_{k} \exp (j 2 \pi k \Delta f t)
$$

Where $\mathrm{X}_{\mathrm{k}}$ is symbol data, $\mathrm{N}$ is size of fft OFDM, and 0 is frequency spacing. The effect of multipath channel is able to be presented as channel impulse response, $H(\mathrm{t})$.

Entrance signal to receiver can be implied as follow formula:

$$
y(t)=\sum_{k=0}^{N-1} H_{k} X_{k} \exp (j 2 \pi k \Delta f t)+z(t)
$$

Table 1. Parameter of simulation OFDM

\begin{tabular}{|l|l|}
\hline OFDM Parameters & Specification \\
\hline Bandwidth, $\mathrm{B}$ & $5 \mathrm{kHz}$ \\
\hline Minimum carrier frequency $f_{0}$ & $9.94 \mathrm{kHz}$ \\
\hline Sampling frequency, $f_{\mathrm{s}}$ & $5 \mathrm{kHz}$ \\
\hline Source Coding & DCT, wavelet \\
\hline Modulation & BPSK \\
\hline Channel Coding & Hamming \\
\hline Symbol per Frame, $\mathrm{N}_{\mathrm{d}}$ & 8192 \\
\hline Total Carrier & 256 \\
\hline Carrier Spacing, $\Delta f$ & $20 \mathrm{~Hz}$ \\
\hline Block Duration, $\mathrm{T}=1 / \Delta f$ & $50 \mathrm{~ms}$ \\
\hline Block Per Frame, $\mathrm{N}=\mathrm{N}_{\mathrm{d}} / \mathrm{K}$ & 32 \\
\hline Guard Time, $\mathrm{T}_{\mathrm{g}}(\mathrm{ms})$ & $16 \mathrm{~ms}$ \\
\hline
\end{tabular}


Channel used in this simulation is characteristic result as presented in the second chapter. Process of generation of multipath channel is started with reference to pattern generation of random log normal with average value 1,028 and variance of 1.35 . The Channel is generated and convoluted with the output of a transmitter, and added with Noise Gaussian. Parameter of channel showing simulation conditions is stationary, without any relative motion between transmitter and receiver. The Noise environment is expressed as $\mathrm{z}$ (t) with Gaussian distribution.

Low-pass equivalent or baseband form of the received signal can be expressed in the following equation:

$$
y(n)=\sum_{k=0}^{N-1} H_{k} X_{k} \exp (j 2 \pi k n)+z(n)
$$

The next process on the receiver is the inverse of the transmitter. After the process of OFDM, continued with demodulate to produce a row binary sequence. The process of decoding (DCT or wavelet) adjusts with the transmitter to produce the output such as the image recovery.

\section{PERFORMANCE EVALUATION OF IMAGE TRANMISSION}

Comparison performance of source coding between DCT and wavelet has conducted by looking curve PSNR that determines the quality of image. Figure 5 and 6 are curve of BER and PNSR as results of image transmission on underwater channel. Line signed - * - is curve of BER and PSNR to transformation DCT with a block $8 \times 8$, while with sign $\square$ - is curve BER and PSNR as results of transformation wavelet.

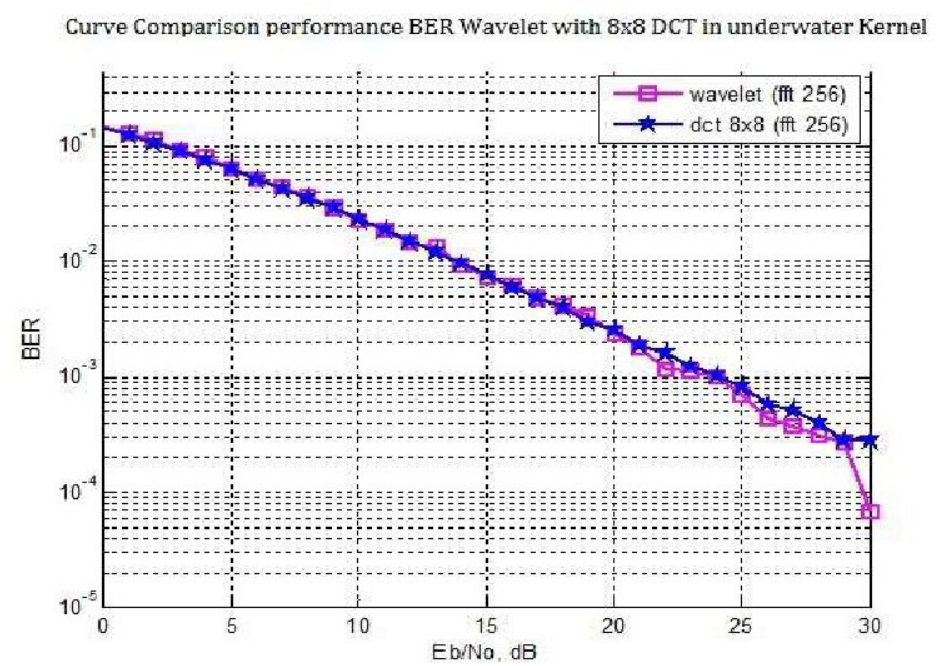

Figure 5. Comparison performance of error rate as function $\mathrm{Eb} / \mathrm{No}$ 
Curve BER shows when Eb/No $20 \mathrm{~dB}$, image with accepted DCT transformation has error 0.0025 , while image transformed using Wavelet has lower error namely 0.0022 . When Eb/No $30 \mathrm{~dB}$ error at received image using DCT transformation as 0,00025 while when using Wavelet transformation as 0,00020 .

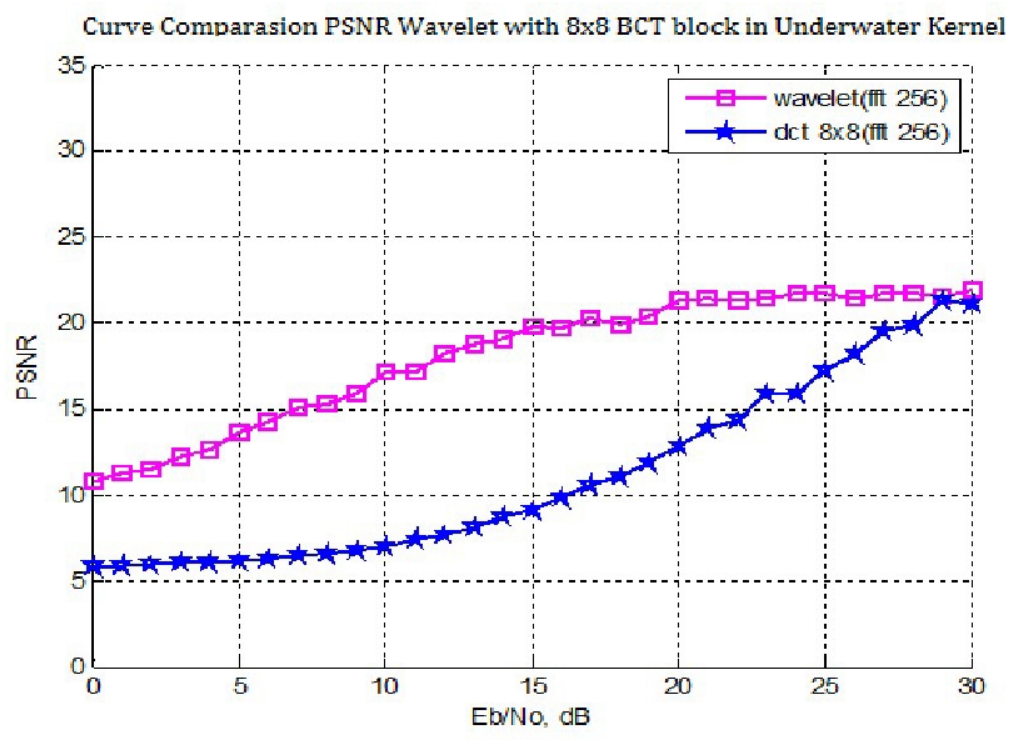

Figure 6. Comparison between PSNR Vs Eb/No

The comparison performance PSNR as a function Eb/No is able to seen as figure 6. When $\mathrm{Eb} / \mathrm{No}$ valued $0 \mathrm{~dB}$, the PSNR wavelet value is higher than DCT with rate $10.77 \mathrm{~dB}$. The Wavelet Transformation is constant at $\mathrm{Eb} / \mathrm{No} 20 \mathrm{~dB}$, while curve of DCT transformation increase continuously till $\mathrm{Eb} /$ No $30 \mathrm{~dB}$. The image can be perfectly reconstructed when PSNR value is $20 \mathrm{~dB}$. PSNR image with Wavelet transformation as $20 \mathrm{~dB}$ when Eb/No 15 $\mathrm{dB}$, while its result from transformation DCT valued $20 \mathrm{~dB}$ when Eb/No 28 $\mathrm{dB}$.

Comparison of the visual image 'bunaken_010.jpg' using source coding technique DCT and Wavelet is shown in Table 2. On the table, it can be seen that at the Eb/No $10 \mathrm{~dB}$, image of Wavelet transformation results can be reconstructed even though there is still noise. While the results of the DCT transformation, at similar Eb/No, the image can't be reconstructed, indicated by number of noise on the image. In the Eb/No $20 \mathrm{~dB}$, the image of DCT transformation results began to show shape of the image even still any noise. The image of the Wavelet transformation in the Eb/No $20 \mathrm{~dB}$ can be seen clearly.

The Value of PSNR is produced by wavelet technique higher than DCT technique. It was because of wavelet work by maintaining coefficient with low frequency to maintain their characteristic of image. While high-frequency was minimized. That makes wavelet that capable for working at worse channel. But, when channel in good conditions, DCT can produce image with 
more sharp and clear pixel than wavelet. It can be observed as in value $\mathrm{Eb} /$ No of $30 \mathrm{~dB}$.

Table 2. Comparison of image visual of result source coding DCT and Wavelet at underwater acoustic channel

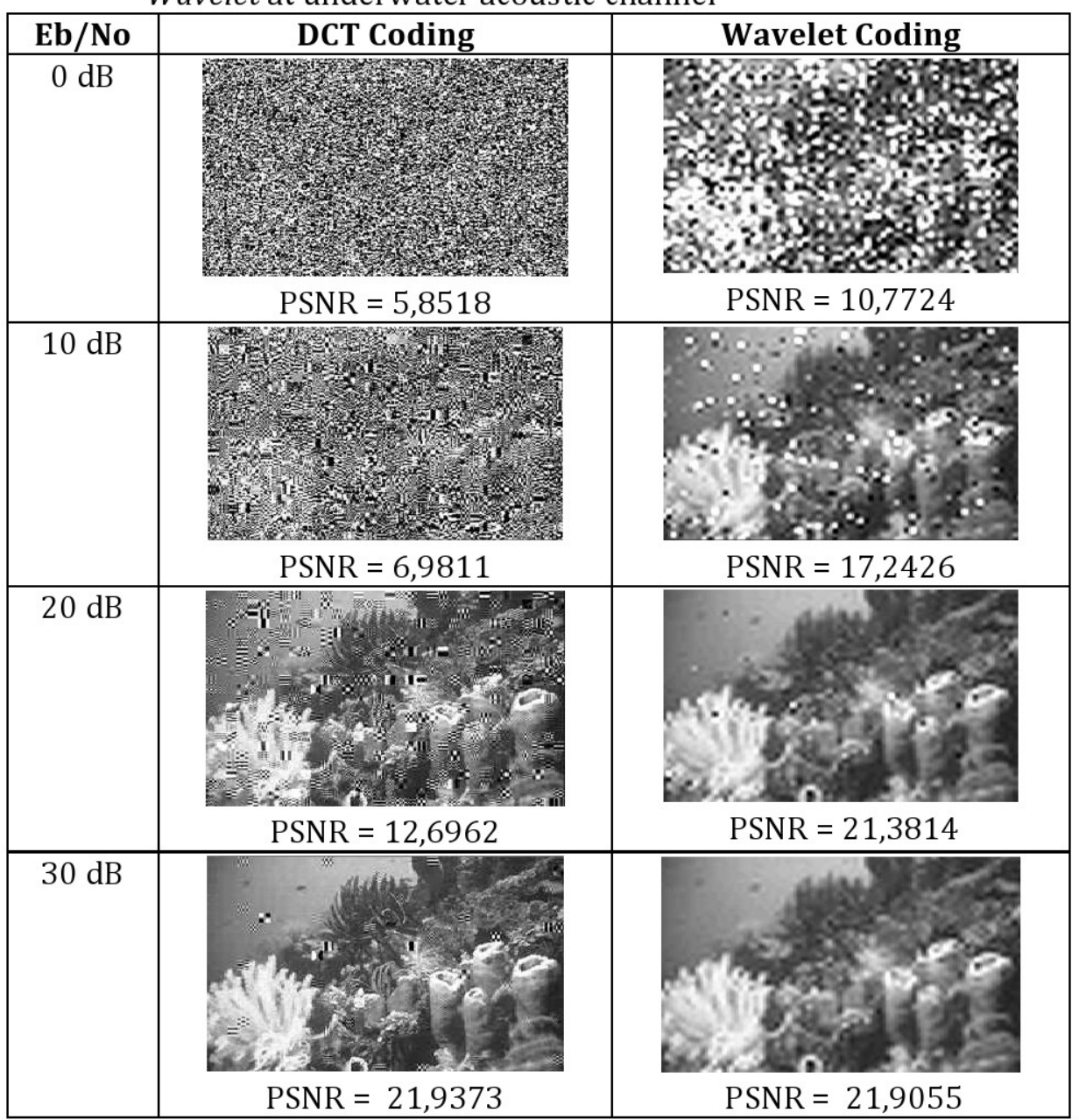

\section{CONCLUSION}

Based on the results of image transmission evaluation over underwater acoustic channel in the swallow-water tropical environment has been done over simulations, image can be acquired as follows.

On image transmission with the DCT transformation cannot be reconstructed well at the low Eb/No value. It is proved by the results of visual image that is worse when the $\mathrm{Eb} / \mathrm{No} 0 \mathrm{~dB}$, and it can be predicted from the low value of PSNR, $5.8518 \mathrm{~dB}$. There are difference at Wavelet transformation that is able to construct image form at Eb/No $0 \mathrm{~dB}$, capable of achieving up to $10,7724 \mathrm{~dB}$. Although there is a lot of noise, image in Wavelet transform still shows a good shape on the image recovery results. 
The use of DCT techniques will give a good performance compared with Wavelet for the channel condition which has a height relative value of $\mathrm{Eb} / \mathrm{No}$, for example, $30 \mathrm{~dB}$.

In the future research, it will be conducted over image transmission modeling with characteristics of frequency selective fading. Among the subcarrier with in the OFDM system, it is possible to use the different modulation and different channel coding techniques. By this strategy, the system is expected able to increase the efficiency of energy transmission.

\section{Acknowledgment}

We would like to say lot of thanks to Laboratorium Hidrodinamika Indonesia (LHI) which has provide facilities for measurement process of propagation parameter of underwater acoustic. So, the research progress of image transmission over underwater acoustic channel has been completed.

\section{REFERENCES:}

[1] Milica Stojanovic, James Priesig, Underwater Acoustic Communication Channels: Propagation Models and Statistical Characterization, IEEE Communciation Magazine, January 2009.

[2] Parastoo Qarabaqi, Milica Stojanovic, Statistical Modeling of a Shallow Water Acoustic Communication Channel, Proc. of Underwater Acoustic Measurements Conference, Nafplion, Greece, June 2009.

[3] Doosti Aref, B. Abbasi Arand, Design and Simulation of a New Model for Shallow Water Multipath Acoustic Channel in the Persian Gulf, The $5^{\text {th }}$ Internaional Symposium on Telecommunications (IST'2010), 2010.

[4] Mandar Chitre, A High-Frequency Warm Shallow Water Acoustic Communications Channel Model and Measurements, Journal of Acoustical Society of America, Vol. 122, No. 5, November 2011, pp. 2580-2586.

[5] Brian Borowski, Characterization of a Very Shallow Water Acoustic Communication Channels, Proc. of OCEANS09, MTS/IEEE BiloxyMarine Technology for Our Future: Global and Local Chalenges, 2009.

[6] Qing-Zhong Li, Wen-Jin Wang, Low-bit-rate coding of underwater color image using improved wavelet difference reduction, Journal of Visual Communication and Image Representation, Vol. 21, pp. 762769, 2010.

[7] Iglesias, I., Song, A., Garcia, J.F., Badiey, M., and Arce, G.R., Image Transmission Over the Underwater Acoustic Channel via Compressive Sensing", The 45th Annual Conference on Information Sciences and Systems (CISS), 23-25 March, 2011.

[8] Beatrice Tomasi, Laura Toni, James Preisig, A Study on the SPIHT Image Coding Technique for Underwater Acoustic Communications, WUWNet 2012, December 2011. 
[9] Zhu Weiqing, Zhu Min, Wang Junwei, Huang Haiyun, Yang Bo, Xu Lijun, Zhao Liang, Signal Processing for High Speed Underwater Acoustic Transmission of Image, OCEANS 2007.

[10] Tri Budi Santoso, Endang Widjiati, Wirawan, Gamantyo Hendrantoro, Parameter Measurement of Acoustic Propagation in The Shallow Water Environment, Proc. of Conference on Information Technology and Electrical Engineering (CITEE) 2012, , Jogjakarta, Indonesia, July 23. 